\title{
Relationship between the topological skeleton, current concentrations, and 3D magnetic reconnection sites in the solar atmosphere
}

\author{
R. C. Maclean ${ }^{1}$, J. Büchner ${ }^{2}$, and E. R. Priest ${ }^{1}$ \\ 1 Institute of Mathematics, University of St Andrews, The North Haugh, St Andrews, Fife, Scotland, KY16 9SS, UK \\ e-mail: rhonam@mcs.st-andrews.ac.uk \\ 2 Max-Planck-Institut für Sonnensystemforschung, Max-Planck-Str. 2, 37191 Katlenburg-Lindau, Germany
}

Received 12 September 2007 / Accepted 26 September 2008

\begin{abstract}
Aims. The aim of this work is to determine the relationship between the 3D structure of the coronal magnetic field, diagnosed by the topological skeleton, and current concentrations as potential sites of 3D reconnection.

Methods. We utilised the results of 3D numerical MHD simulations of an observed EUV bright point (BP) in the solar atmosphere. The simulations are based on MDI line-of-sight magnetogram data from 13 June 1998. We analysed the results of the simulations using the method of magnetic charge topology. Three different methods of reducing the magnetogram to a set of point magnetic sources are tested.

Results. Observations of the BP show a rotation of one of its main magnetic source regions. Numerical simulations of this rotational motion result in a localised build-up of parallel electric current, which is dissipated by anomalous resistivity, causing 3D magnetic reconnection and BP heating. The magnetic topological structure of the simulated BP was also calculated, and a portion of the topological separatrix surface bounding the magnetic flux of the rotating source region is found to correspond to the locations of current build-up and heating. All three magnetogram reduction methods produce similar results for the large-scale magnetic field structure.

Conclusions. Magnetic topology is a useful method for predicting the locations of coronal current concentrations, insofar as the results of our simulations show that strong integrated parallel electric fields are found only along topological separatrix surfaces. However, further investigation is necessary to determine exactly which parts of the reconstructed separatrices will host the electric currents. Topological magnetic field reconstructions also cast light on the location of coronal BP heating, which occurs as a result of the dissipation of the currents by 3D reconnection. The choice of the magnetogram reduction algorithm does not greatly affect the large-scale topological features of the resulting reconstructed magnetic field. Further work is required to compare these results with data for other observed BPs.
\end{abstract}

Key words. Sun: atmosphere - Sun: corona - Sun: magnetic fields - magnetohydrodynamics (MHD) - methods: numerical

\section{Introduction}

Topological properties of the coronal magnetic field such as separators and separatrices, and geometrical features such as quasi-separatrix layers, are potential sites for magnetic reconnection and heating in the solar atmosphere, because electric currents can easily build up along them (Priest \& Démoulin 1995; Démoulin et al. 1996; Priest \& Titov 1996; Priest et al. 2005; Büchner 2006a). Coronal currents indicate that excess energy is stored in the magnetic field and, according to present theory, can be released by collisionless dissipation (e.g. Shay et al. 1998; Büchner \& Elkina 2006; Büchner \& Daughton 2007). Once a certain critical drift velocity of the current carriers is attained collisionless dissipation starts and reconnection becomes possible (e.g. Büchner 2007). Consequences can be a heating of the corona, the acceleration of particles to high energies and the formation of plasma jets (e.g. Yokoyama \& Shibata 1996). Different methods have been proposed to model the geometrical and topological features associated with the formation of current concentrations. This includes the concept of quasi-separatrix layers (QSLs) (Titov 1999; Titov et al. 2002) as well as the magnetic charge topology (MCT) method (Longcope 1996; Brown \& Priest 1999).

Coronal bright points (BPs) were first observed by Vaiana et al. (1970). They appeared to be closely related to bipolar magnetic features (e.g. Golub et al. 1977). This lead to the development of reconnection scenarios of bright point heating, first in two dimensions (e.g. Priest et al. 1994). Later three-dimensional reconnection models were suggested. In order to verify $3 \mathrm{D}$ reconnection near BPs it is appropriate to analyse BP related magnetic fields using appropriate analysis methods of the field geometry and topology. Here we apply the MCT method to the case of a coronal bright point which was simulated by Büchner et al. (2004) based on observations by Brown et al. (2001). SoHO/MDI and TRACE both observed a bright point from its birth around 20:00 UT on 13 June 1998 until its disappearance around 16:00 UT the next day. These observations were used as a starting point for 3D numerical MHD simulations of the plasma and magnetic field in the solar atmosphere. The observed rotation of one of the magnetic sources (the main negative polarity rotated $\pi / 2$ radians clockwise over the course of $2 \mathrm{~h}$ from 09:41 UT) was applied as a boundary condition and compared 
to the results of the simulated consequences of rotating the other main source region. The results of the simulation give valuable clues about which parts of the magnetic field are most important in determining the behaviour and evolution of the bright point.

In modelling the coronal potential magnetic field caused by an observed normal magnetic field at the photosphere, a choice of approaches is available. First, one could regard the photospheric field as continuous (the QSL method). With the magnetic field values at every point on a spatial grid one could then obtain both the skeleton (of separatrices) and a quasi-skeleton (of quasiseparatrix layers), which is quite difficult to do to high accuracy. Or, one could approximate the photospheric field by a series of point sources (the MCT method). In such approach the quasinulls (regions of weak magnetic fields) and quasi-separatrices of the QSL approach become exact nulls and separatrices. The strengths and weaknesses of both approaches were discussed by (Démoulin et al. 1996) and the choice of method will depend on the aim and purpose of the modelling effort, as well as what type of feature is being modelled.

In fact, the QSL method was developed in response to a limitation of the MCT method (for details, see Démoulin et al. 1996). In an MCT field reconstruction, there will exist about the same number of photospheric null points as the number of point sources. This is an artefact of the model and does not mean that all these photospheric nulls will exist in the real field; in fact it is highly unlikely that a real null would be sitting exactly in the photospheric plane. Fields constructed using the QSL method replace these photospheric nulls and their separatrix surfaces (across which the fieldline connectivity is discontinous) with quasi-separatrix layers. These are thin layers in which the fieldline connectivity gradient is very steep but not discontinuous. This is likely to be a more accurate representation of the real magnetic field on the Sun. In fact, in the real coronal magnetic field, it is likely that both QSLs and true separatrix surfaces exist together. Some true magnetic null points might still exist in the solar atmosphere high above the photosphere, and each of these nulls is associated with a true separatrix surface. For these reasons, the QSL method is often the better choice.

However, reconnection and heating may occur both at sites of enhanced current concentrations for which separators and separatrices are indicative as well as quasi-separators and quasiseparatrices. The main aim of this work is to determine to which degree enhanced current concentrations are related to the separatrix positions as they can be obtained by the MCT method which is simpler, even though it produces some spurious nulls. Furthermore, it is often the case that the main magnetic flux in the photosphere is given in the form of discrete fragments (each made up of several MDI pixels but separated by tens of pixels). In this case the MCT method is very much appropriate. Of course, the sources could be given also with finite size, but, again for simplicity, we choose to adopt a point-source model.

The paper is structured as follows. In Sect. 2 we describe the methods used for the numerical simulation and give an overview of the technique of magnetic topology and the assumptions we have used to construct the topological models. Section 3 contains a topological analysis of the initial magnetic structure of the bright point region. The main results are in Sect. 4 where we look at the topological consequences of rotating the main magnetic source regions of the bright point, and how that matches up with the observed locations of brightenings in the corona. Then in Sect. 5 we deal with the question of the robustness of the topological model we are using and how it is affected by the level of accuracy chosen. Finally in Sect. 7 we present and discuss our conclusions.

\section{Modelling techniques}

\subsection{Numerical simulations}

A 3D numerical MHD code was used to simulate the plasma in the photosphere, chromosphere, transition region, and corona. A full description of the code can be found in Büchner et al. (2005); here we give a short overview of its essential features. The numerical simulation approach consists of three parts. First, the observed longitudinal (line-of-sight) photospheric magnetic field component is used to obtain an initial three-dimensional force-free magnetic field configuration. An appropriate extrapolation method, compatible with the requirements of the boundary conditions of MHD simulation boxes, is described in Otto et al. (2007). The essence of this method is that at the boundaries the horizontal components $\left(B_{x}\right.$ and $\left.B_{y}\right)$ are related to the vertical component $B_{z}$ through fulfilling the force free condition (3), and at the same time, fulfilling $\nabla \cdot \boldsymbol{B}=0$. For this sake a periodicity in the horizontal $(X$ and $Y$ ) directions is assumed to obtain a solution in a non-periodic region which is a quarter of a fully periodic one. For a solution in the domain $0 \leq x \leq L_{x}, 0 \leq y \leq L_{y}$ the fully periodic region would be $-L_{x} \leq x \leq L_{x},-L_{y} \leq y \leq L_{y}$. Within the latter the total magnetic flux is balanced to a high degree. Since the solution of MHD equations, however, requires a locally symmetric boundary condition for the current as well as for the plasma density which should not create an artificial discontinuity in the field-aligned current flows. To meet this condition we use instead a Green's function approach which satisfies line-symmetric boundary conditions. Using this extrapolation technique the simulation model is initialised with a Fourier-filtered photospheric magnetic field, based on MDI observations of the longitudinal components of the solar magnetic field. The filtering is applied since the numerically stable solution of the partial differential MHD equations requires a sufficient resolution of magnetic field gradients by the finite grid distances (about 8-16 grid points resolving the shortest wavelength of the spatial Fourier modes). Without additional information about the photospheric currents or vector magnetic fields, we assume in the beginning, at $t=0, \alpha=0$ i.e. we start with a potential field extrapolation.

Because the plasma- $\beta$ is small throughout the chromosphere and corona, the physics of the solar atmosphere is determined by the magnetic field and not by the plasma pressure. Hence, we can just "fill" the extrapolated magnetic field configuration with plasma. We choose to start with an initial density and temperature stratified with height following the equilibrium VAL model of Vernazza et al. (1981) in its version "C". Deviating from VAL we assume, however, somewhat smaller values for the plasma density in the lower chromosphere, limiting it to 100 times the coronal density. This is done partly for numerical reasons because larger densities correspond to larger collision frequencies which would require significantly smaller simulation time steps than desirable for large scale three-dimensional simulations. This limitation of the density is justified since we do not draw special attention to the processes in the lower chromosphere, we just want to ensure a sufficiently strong coupling between the neutral gas and plasma. Further, in order to resolve the transition region well (by at least ten grid points, see Sect. 2.1), we enhanced the width of the transition region artificially to about $1500 \mathrm{~km}$. For the side boundaries of the simulation box, we assume the same line symmetry across the $X$ and $Y$ boundaries for plasma density and the density of a neutral gas component, which we also take into account, as well as for the velocities and currents. At the lower (photospheric) boundary the tangential velocity is defined by strongly coupled plasma 
and neutral gas motion, and the boundary normal velocity is set to zero (no emerging or submerging flux). The normal magnetic field uses $\nabla \cdot \boldsymbol{B}=0$ and the horizontal components are computed from $\nabla \times \boldsymbol{B}=\alpha \boldsymbol{B}$. Density and pressure are assumed symmetric (zero normal derivative). At the top (coronal) boundary symmetric conditions (zero normal derivatives) are assumed. Based on the changes of the photospheric magnetic field, a consistent horizontal velocity profile is imposed as a boundary condition at the simulation. In order to avoid plasma emergence and submergence we apply a vortex motion satisfying $\nabla \cdot \boldsymbol{u}_{n}=0$, where $u_{n}$ is the neutral gas velocity. This way the density is conserved. It is contained in the $X, Y$ directions so it can be expressed as a potential function $U$ with $\boldsymbol{u}_{n}=\nabla \times\left(U \boldsymbol{e}_{z}\right)$. The motion is imposed for the neutral gas throughout the whole simulation box. However only in the chromosphere, where the collision frequency is sufficiently large, it effectively couples to the plasma.

We solve the following set of MHD equations:

$$
\begin{aligned}
\frac{\partial \rho}{\partial t}= & -\nabla \cdot \rho \boldsymbol{u}-\mu\left(\rho-\rho_{\mathrm{e}}\right), \\
\frac{\partial \rho \boldsymbol{u}}{\partial t}= & -\nabla \cdot\left[\rho \boldsymbol{u} \boldsymbol{u}+\frac{1}{2}\left(p+B^{2}\right) \underline{\underline{1}}-\boldsymbol{B B}\right] \\
& -\mu \rho\left(\boldsymbol{u}-\boldsymbol{u}_{\mathrm{e}}\right),
\end{aligned}
$$

$$
\begin{aligned}
\frac{\partial \boldsymbol{B}}{\partial t}= & \nabla \times(\boldsymbol{u} \times \boldsymbol{B}-\eta \boldsymbol{j}), \\
\frac{\partial h}{\partial t}= & -\nabla \cdot h \boldsymbol{u}-\frac{(\gamma-1)}{\gamma} h^{1-\gamma}\left(2(\gamma-1) \eta \dot{j}^{2}\right) \\
& +\frac{(\gamma-1)}{\gamma} h^{1-\gamma} \mu\left(h-h_{\mathrm{e}}\right) .
\end{aligned}
$$

In Eqs. (1)-(4) the subscript e denotes the equilibrium variables. In the energy Eq. (4) the pressure $p$ is substituted by the internal energy $h=(p / 2)^{1 / \gamma}$ which yields a continuity equation for $h$ in the absence of energy sources and sinks. For the polytropic index we use the adiabatic law $\gamma=5 / 3$. An important property of the solar atmosphere is the strong collisional coupling between the plasma and the neutral gas in the photosphere and chromosphere. There collisions cause friction between the plasma and the neutral gas, energy exchange, and also lead to ionization and recombination. In Eqs. (1)-(4) we use the same effective frequencies for the ionization, recombination, friction and energy exchange processes, because the sole purpose of the introduction of these collisions is to maintain a strong coupling between plasma and the neutral gas below the transition region and to let the coupling vanish in the transition to the corona, thereby maintaining the transition region itself. In this sense and for this purpose, the exact values of these frequencies do not matter as long as they are sufficiently large $(\mu \gg 1)$. We did not include radiative cooling and thermal conduction processes, this is beyond the scope of the present paper, where we focus on the formation and location of current concentrations in the corona.

We solve the partial differential MHD Eqs. (1)-(4) by means of a finite difference approximation. We apply a Leapfrog scheme which is second order in accuracy and very low in numerical dissipation. As usual in schemes, based on the FluxCorrected Transport (FCT) paradigm, un-physical oscillations are damped on the grid scale. Second order derivatives are treated with a Dufort-Frankel scheme allowing the consideration of small resistivities. We choose a non-uniform grid to meet the requirement of a high plasma- $\beta$ (higher plasma/gas pressure than magnetic pressure) at the lower boundary reaching

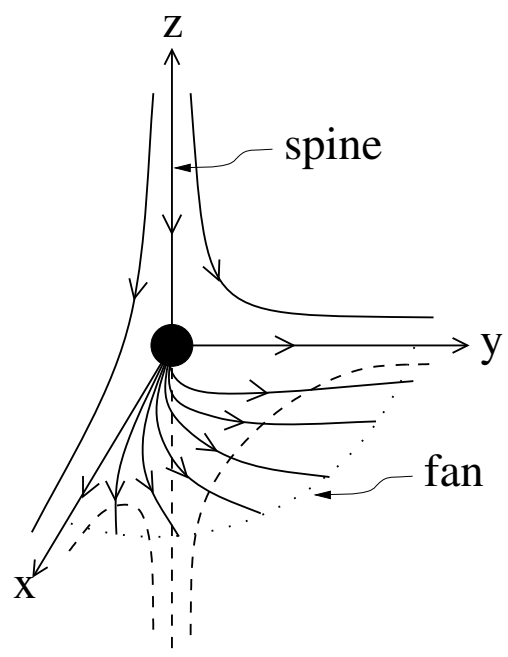

Fig. 1. Structure of a first-order generic potential magnetic null point.

the much lower- $\beta$ corona via the low- $\beta$ chromosphere. We also choose a non-uniform grid to be able to spatially resolve the chromosphere and the steep gradients in the transition region well enough. Our grid has 49 points in the vertical direction $(Z)$, and 131 points in each of the horizontal (periodic) $X$ and $Y$ directions.

The code was verified, e.g., by investigating the bulk plasma acceleration due to perpendicular electric fields based on Soho observations on October 17, 1996 (Büchner et al. 2005) and in preparation of the Hinode data analysis (Büchner 2007).

\subsection{Overview of magnetic topology}

Studying the magnetic topology of a magnetic field is an excellent way to extract vital information about the shape and connectivity of that field, reducing it to only its essential structural features. For this work, we decided for simplicity to reduce the magnetograms to point magnetic sources and use potential magnetic fields. Each point magnetic source represents a concentration of positive or negative flux in the magnetogram. The integrated flux corresponds to the strength of the point source, which is located at the centre of mass of the flux concentration; flux concentrations with a peak value below $50 \mathrm{G}$ are neglected. Brown \& Priest (2000) showed that potential fields are a good approximation to the real coronal field in cases where the nonlinearity in the field is not too severe and hence the current remains relatively low, which holds in quiet Sun regions like the one we study here. Although the exact shape (geometry) of the real field may differ from our results, the important topological features will be the same.

The analysis begins by finding the locations of the magnetic null points of the field, where $\boldsymbol{B}=0$. These can be either in the photosphere (on the base of our 3D simulation box) or in the corona (within the 3D volume). Figure 1 shows the structure of the magnetic fieldlines near a first-order generic potential null point, i.e. the type of null found in the current scenario. The structure can be found by linearising the expression for $\boldsymbol{B}$ near the null and using the three eigenvalue-eigenvector pairs to give starting points for extrapolation of the spine and fan fieldlines (Parnell et al. 1996). A null is called positive if its fan fieldlines are directed outwards and negative otherwise. For a null in the photosphere, it is called prone if its spine fieldline lies in the photosphere and upright otherwise. 
When many magnetic sources are present, the number of nulls in a topology will also be large. The exact number is governed by two Euler characteristic equations. In the photosphere,

$S+n_{\mathrm{u}}=n_{\mathrm{p}}+2$,

where $S$ is the number of magnetic sources, $n_{\mathrm{p}}$ is the number of prone nulls and $n_{\mathrm{u}}$ is the number of upright nulls. In the corona,

$S_{+}-n_{+}=S_{-}-n_{-}$,

where $S_{+/-}$is the number of positive/negative magnetic sources and $n_{+/-}$is the number of positive/negative nulls. Note that for these equations to hold, the total flux from the sources must be in balance; this condition is generally fulfilled by the addition of a "balancing" source at infinity. These equations provide a useful check that all nulls in the topology are likely to have been detected.

Extrapolation of all the spine and fan fieldlines is the next step in the topological analysis. The fieldlines of the fans extend out to form curved surfaces called separatrix surfaces. Where two such separatrices intersect, a separator fieldline is formed, which joins the two null points. Both separatrices and separators define the boundaries between regions of different magnetic connectivity in the topology. This makes them prime locations for magnetic reconnection to take place (Priest et al. 2005). In Sects. 3 and 4, we construct the initial magnetic topology of the bright point region just as described here, and use our assumptions about the motion of the sources to predict on which separatrix surfaces signatures of reconnection should be observed.

\subsection{Assumptions in the topological models}

Before this topological modelling can commence, the magnetogram must be reduced to a set of point magnetic sources. In this bright point investigation, we experiment with three different methods of producing the set of sources from a magnetogram. Section 5 describes how analysis of the results of all three methods can determine to what extent the calculated topology is affected by the method of picking point sources. Here, we simply explain the actual methods used.

Two of the methods involve Fourier filtering. The magnetogram data is Fourier-filtered to either 8th or 16th order. This means that only lower-order terms are included, so only magnetic field sources that are spatially relatively large are detected, automatically getting rid of the small-scale fluctuations. This technique picks out the regions of strongest "signal", i.e. strongest line-of-sight magnetic flux, and each region is represented by a point source, with strength proportional to the magnetic flux of the region. 16th-order Fourier filtering is more accurate and so picks out many more source regions than the 8 th-order method does. Any source with strength less than $50 \mathrm{G}$ is neglected. This threshold value may seem high compared to a typical noise level for the MDI instrument. However, the choice of threshold is always a compromise between values so high that only a handful of source regions are identified and important information about the magnetic field configuration is lost, and values so low that you are down in the noise and the selected "source regions" become meaningless. In this case, the choice of $50 \mathrm{G}$ was made in order to select a reasonable number of the strongest magnetic features visible in the vicinity of the bright point.

In the third alternative method, the raw magnetogram data is smoothed to get rid of small-scale numerical fluctuations which would introduce tiny spurious sources. Each point magnetic
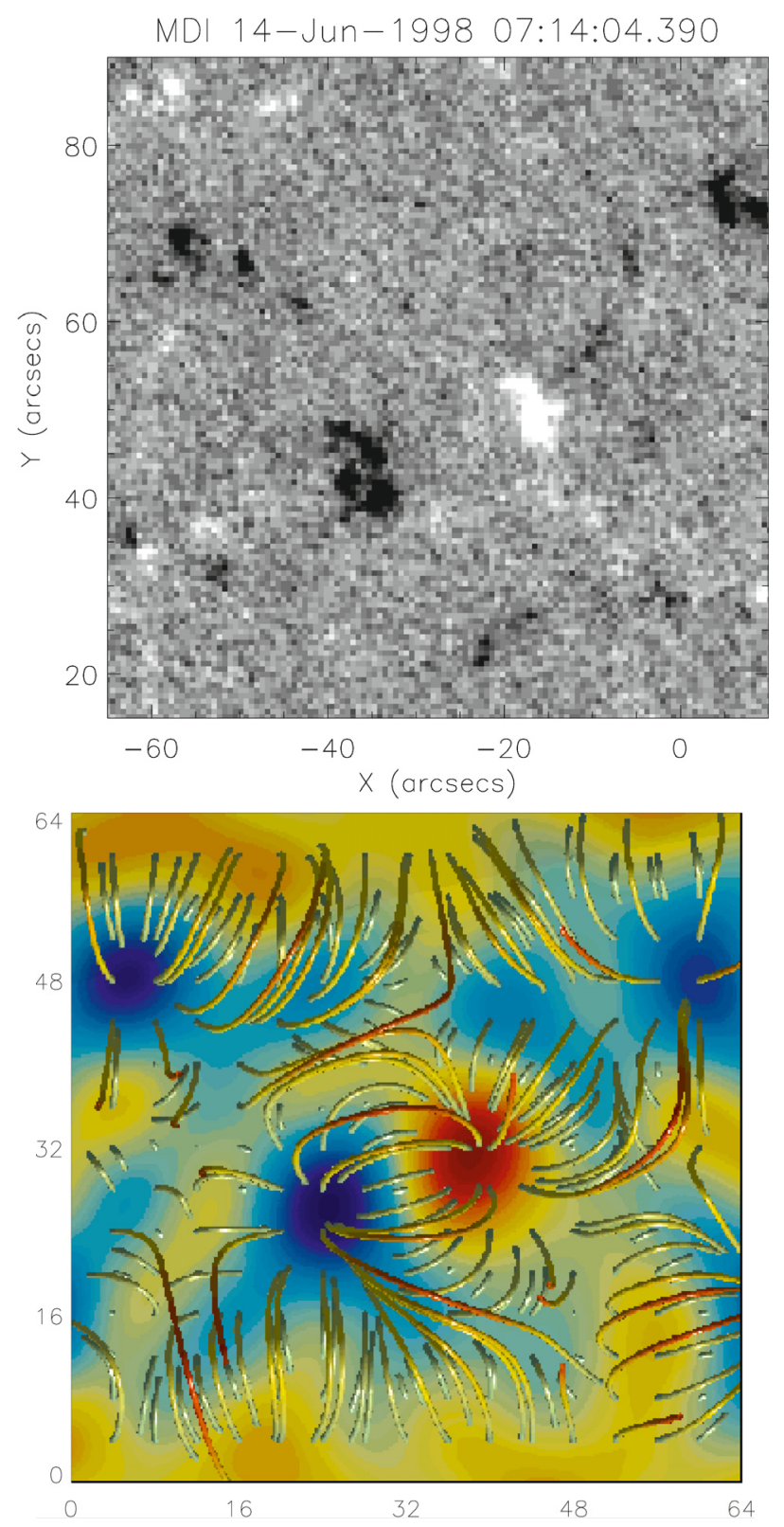

Fig. 2. Initial photospheric magnetic field. Above: raw MDI magnetogram, with greyscale shading indicating magnetic field strengths between \pm 50 G. Below: eight Fourier-mode-filtered, with red/blue representing positive/negative line-of-sight magnetic field. The overlaid magnetic fieldlines have been extrapolated for the case of a potential field. Each unit on the axes corresponds to $500 \mathrm{~km}$ on the photosphere.

source represents a concentration of positive or negative flux in the smoothed magnetogram. The integrated flux corresponds to the strength of the point source, which is located at the centre of mass of the flux concentration; again, flux concentrations with a peak value below $50 \mathrm{G}$ are neglected.

\section{Magnetic structure of the bright point region}

Figure 2 shows an MDI magnetogram taken just before the rotation phase of the negative source begins. The bright point itself is located in the corona directly above the two central sources. Some extrapolated potential magnetic fieldlines are shown, which highlight part of the structure of the field. The scale on the axes is in units of $500 \mathrm{~km}$, so that the total box size 
is $32 \times 32 \mathrm{Mm}$ or $75 \times 75^{\prime \prime}$. This scaling is used throughout the paper.

Topological analysis (using the 8th-order Fourier-filtering method) reduces this complex photospheric field to fifteen point magnetic charges, five of which are positive and ten negative. This number of sources is not imposed as part of the model, but is a natural result of the choice of threshold magnetic field value for defining the source regions. Due to the imbalance in the total magnetic flux, we also insert a compensating negative source at infinity. This is a standard and integral part of the MCT model, as flux balance is required for the divergence of the magnetic field to be zero. The source is added at infinity, rather than at some finite distance away from the other sources, to minimise its influence on the topology and avoid bias. The two magnetic sources that represent the BP bipole are the strongest sources in the chosen region, but not by orders of magnitude; all the magnetic charges in the topology are strong enough to be important for the topological structure in some way or another. The BP sources do not dictate the whole magnetic structure of the region. Although they are dominant in the centre of the simulation box, towards the edges of the box the other sources dominate the magnetic field structure.

Fifteen null points are found in this configuration; nine positive and six negative. One of the positive nulls is in the corona and all the other nulls are prone, sitting on the photospheric boundary. Note that the Euler Eqs. (5) and (6) are satisfied since the coronal null has a "mirror image" below the photosphere which is not physically meaningful but must be included in the equations. Extrapolation of the spine and fan fieldlines shows that eighteen separators are present. The photospheric footprint of the topology is shown in Fig. 3.

\section{Simulation of magnetic reconnection}

\subsection{Observed motion: the main negative source rotates}

The photospheric magnetograms of the bright point region show the main negative source $(\mathrm{S} 1)$ rotating about $\pi / 2$ radians clockwise over the course of $2 \mathrm{~h}$ from 09:41 UT. In this section we look at the effects of the magnetic reconnection caused by this rotation, and how they are reflected in the magnetic topology of the region.

Figure 4 is a contour plot of the parallel electric field integrated along the magnetic field, $90 \mathrm{~min}$ after the start of the MHD simulation of the main negative source rotating (Büchner et al. 2004). The parallel electric fields are due to enhanced currents flowing parallel to the magnetic field that exceeded the threshold of anomalous resistivity (Büchner \& Daughton 2007; Büchner \& Elkina 2005).

Strong concentrations of parallel electric field exist in the simulation box along fieldlines that connect to the photospheric surface at locations corresponding to both the positive and negative main sources. There is also a curve of high values of integrated $E_{\|}$extending down and right from the positive source before it twists back around to the left, along the southern edge of the base of the simulation box.

In fact, the position of this curve corresponds very well to the position of an important separatrix surface in the magnetic topology, as shown in Fig. 5. The negative null N1 has a separatrix surface associated with it. This $3 \mathrm{D}$ separatrix forms a dome structure which covers the rotating negative source and encloses all of its flux. The dome is bounded in the photosphere by its own separatrix traces, and more importantly, by the linked spines of several positive nulls (highlighted in orange in Fig. 3).

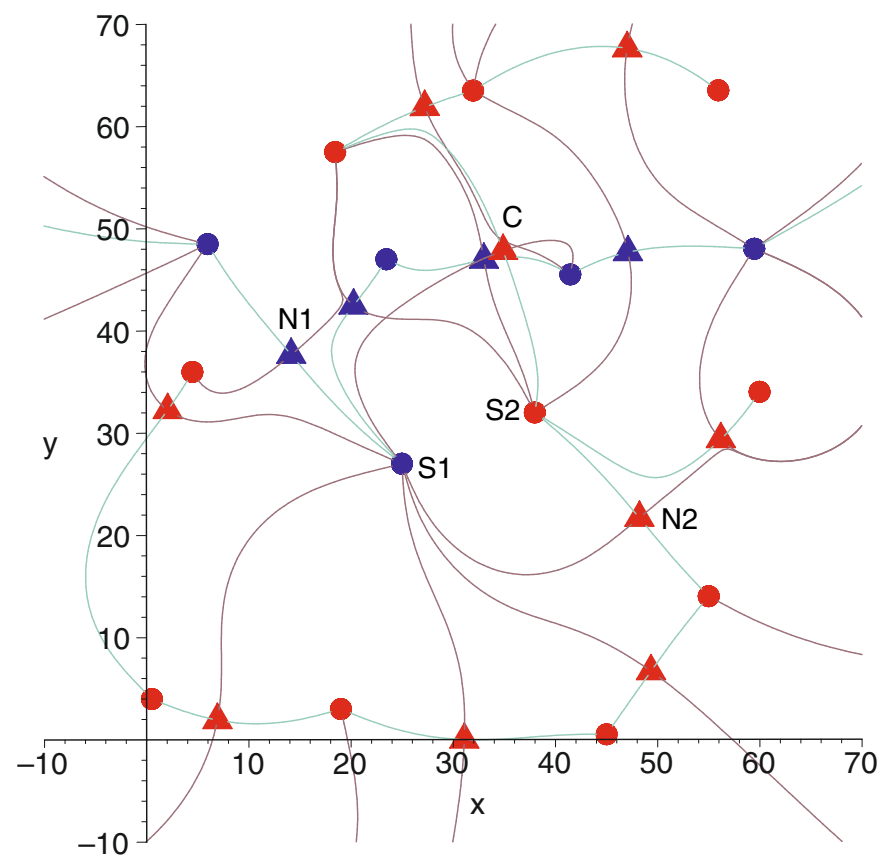

Fig. 3. Photospheric footprint (only photospheric fieldlines shown for photospheric nulls) of the initial magnetic field from Fig. 2. The source locations and strengths were determined using the 8th-order Fourierfiltering method. Positive (negative) sources are red (blue) circles. Positive (negative) null points are red (blue) triangles. Spine fieldlines are green and separatrix fieldlines are purple. The main negative and positive sources of the bright point are labelled S1 and S2 respectively, and the three important nulls are labelled N1, N2 (photospheric), and $\mathrm{C}$ (coronal). Fieldlines in the topological skeleton that mark the photospheric boundaries of the BP's magnetic flux are highlighted: for S1 in orange and for $\mathrm{S} 2$ in pink.

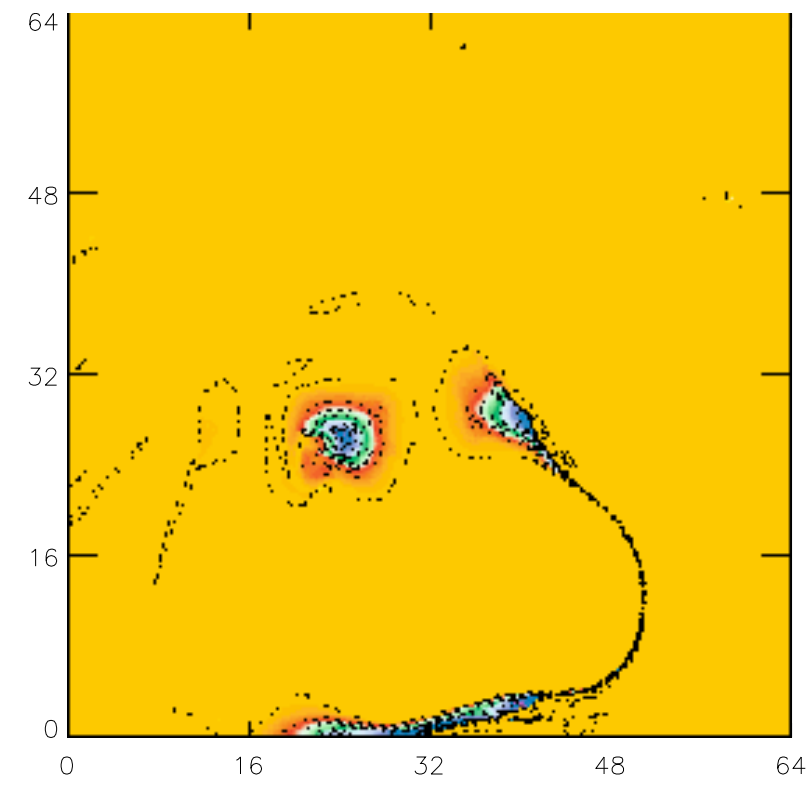

Fig. 4. Contour plot of parallel electric field $\left(E_{\|}\right)$integrated along the magnetic field as obtained from MHD simulation of the main negative source rotating clockwise, shown after 90 min of evolution.

It has not yet been fully explained why only some sections of the photospheric trace "light up" in $E_{\|}$integrated along the magnetic fieldlines, but a possible theory is that, as most of the magnetic flux from the main negative source connects to 


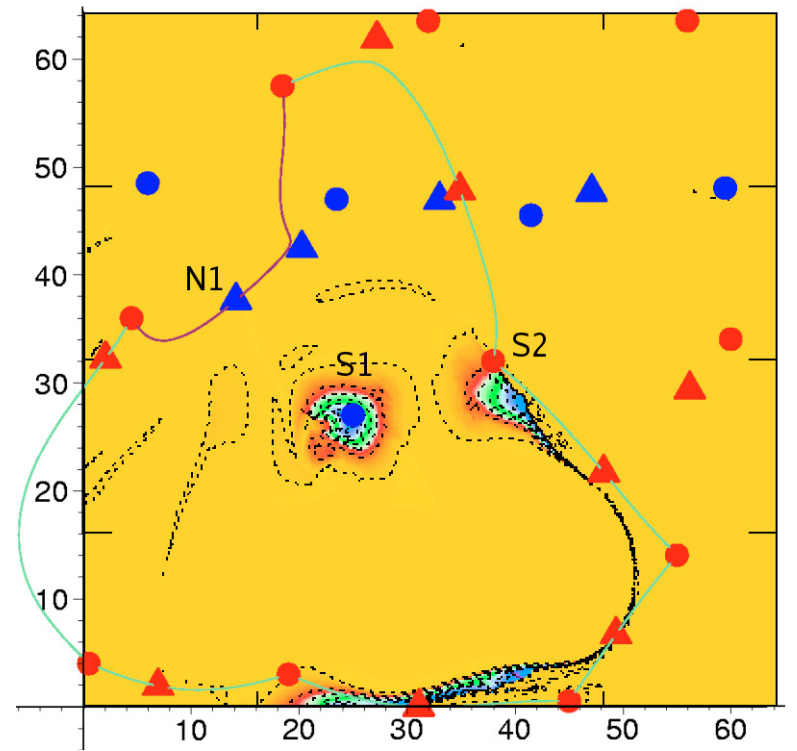

Fig. 5. The photospheric footprint (based on 8th-order Fourier-filtered sources) of the separatrix dome that covers the rotating negative source (S1), and how it compares with the contour plot of $E_{\|}$integrated along the magnetic fieldlines.

the main positive source, most of the stress is felt along these fieldlines. As the clockwise rotation proceeds, some of these fieldlines are forced to reconnect and are pulled out along the separatrix boundary, still highly stressed and associated with large values of integrated $E_{\|}$. In Sect. 5.4 and Fig. 19 we show and discuss the calculated quasi-separatrix layers of the magnetic field from the numerical simulation. It seems that the sections of the topological separatrix surfaces along which QSLs are detected are close to being the same sections along which the high values of integrated $E_{\|}$show up. Therefore it may be that high values of $E_{\|}$integrated along the magnetic fieldlines are associated with strong gradients in fieldline connectivity. This makes sense because we would expect magnetic reconnection to be occurring in just such regions. The QSL model may therefore have more to say than the MCT model about exact locations of reconnection and heating within a (quasi-)separatrix surface. An interesting follow-up, which is outside the scope of the present work, would be to investigate the exact correlation between the location of the QSLs and the location of the regions of high integrated $E_{\|}$.

Figure 6 shows a 3D view of the separatrix dome. As you can see, the line of spines along which it connects to the photosphere is in the same position as the region of high integrated $E_{\|}$. This means that the rotation of the source is causing reconnection and heating along the separatrix surface which encloses it. Although this line of spines will not exist in precisely this form in the real coronal field, and most of the photospheric nulls are spurious, this model still has meaning. The rotation of the negative source will cause fieldlines to reconnect from the main positive source along the boundary where the connectivity changes to each positive source in the line of spines in turn. The magnetogram does not show a line of isolated point sources, but one long continuous source region of positive flux - so in the real field the reconnection and change of connectivity will be continuous rather than discrete, but the model still shows that this is the separatrix surface where the reconnection will take place (although it cannot predict exactly where on the surface the current will be localised).

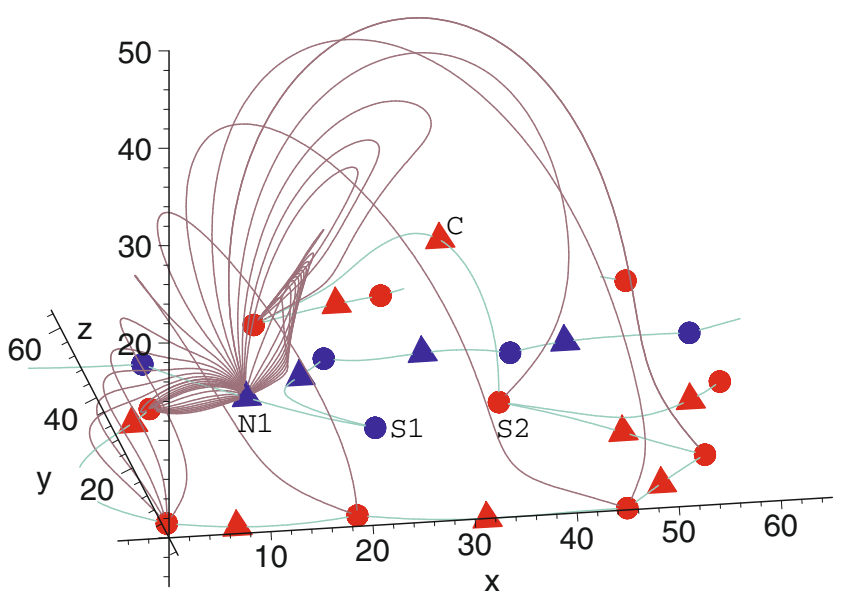

Fig. 6. 3D view of the separatrix dome covering the rotating negative source, with sources calculated using the 8th-order Fourier-filtering technique. To improve ease of visualisation, all spines are shown but only separatrix fieldlines in the separatrix surface under discussion are also displayed. The dome is bounded in the photosphere by the set of green spine fieldlines whose location corresponds to the region of high $E_{\|}$integrated along the magnetic fieldlines.

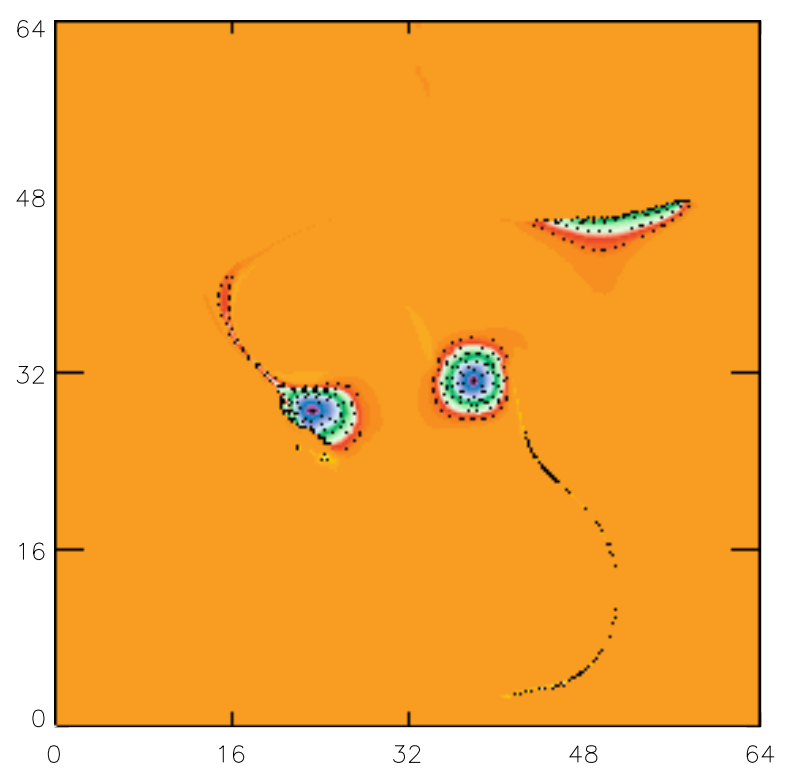

Fig. 7. $E_{\|}$integrated along the magnetic fieldlines as obtained from MHD simulation of the main positive source rotating clockwise, shown $90 \mathrm{~min}$ after the start.

\subsection{Model assumption: the main positive source rotates}

In this section we look at the effect of rotating the main positive source (S2) instead of the negative one (S1). Figure 7 shows the integrated parallel electric field that results, $90 \mathrm{~min}$ into the simulation. There are similarities and differences when this result is compared to the previously discussed simulation of the negative source rotating. In both, there are strong buildups of integrated $E_{\|}$at the sites of the two main sources themselves. However, there are two new regions of high integrated $E_{\|}$in this simulation; one reaching diagonally up and left away from the negative source, and one horizontal strip above and to the right of the positive source. Interestingly, the region from the previous simulation that stretches away from the positive source below and to the right of it still exists in this simulation, although it is the weakest region detected. 


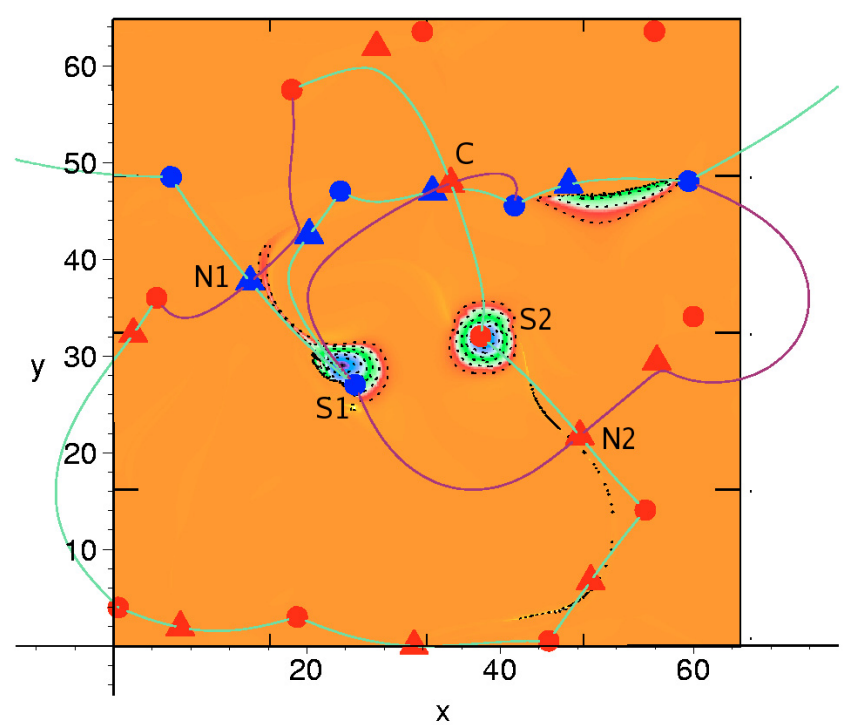

Fig. 8. The photospheric footprint (based on 8th-order Fourier-filtered sources) of the separatrix surfaces that surround the rotating positive source, and how it compares with the contour plot of $E_{\|}$integrated along the magnetic fieldlines. Separatrix covering negative source also shown.

In the previous simulation, the rotating negative source is covered by one separatrix dome whose photospheric traces surround the source. However, the positive source considered here requires not one but two separatrix surfaces to completely surround it. This seems strange at first, but essentially the pointsource model is just a simple way to find out where the changes in fieldline connectivity are. It doesn't matter how many separatrix traces it takes to surround the rotating source in the photosphere, what matters is that it should be these separatrices and no others that show the signature of reconnection (in terms of heightened values of $E_{\|}$integrated along the magnetic fieldlines). Anyway, in this model, one of the separatrices is associated with the positive coronal null point $\mathrm{C}$. This separatrix touches the photosphere all the way along the line of spines of negative nulls running approximately from left to right across the upper part of the footprint (highlighted in pink on the photospheric footprint, Fig. 3). The other important separatrix is that associated with the positive prone photospheric null N2. This separatrix touches the photosphere first along its own purple separatrix traces and then along the connecting spines directly out to the edge of the diagram. The photospheric footprint of these two separatrices is given in Fig. 8, which also shows the contour plot of $E_{\|}$integrated along the magnetic fieldlines for comparison.

In fact, the figure confirms that the two regions of high integrated $E_{\|}$above the sources lie along parts of the two separatrix surfaces that surround the positive source. The region above and to the left of the negative source lies along part of one of the spines that bounds both of the separatrix surfaces in the photosphere. The region above and to the right of the positive source follows almost exactly part of the spine lines of a negative null lying in the footprint of the coronal null's separatrix. A 3D view of these separatrices is shown in Fig. 9.

It is interesting that rotation of the positive source also causes a small buildup of integrated $E_{\|}$along the separatrix surface associated with the negative source. This may be due to the fact that the negative null where this separatrix originates is connected by separators to both the positive nulls whose separatrices surround the positive source. Thus, the surfaces are linked and shear applied to one may have a knock-on effect on the others.

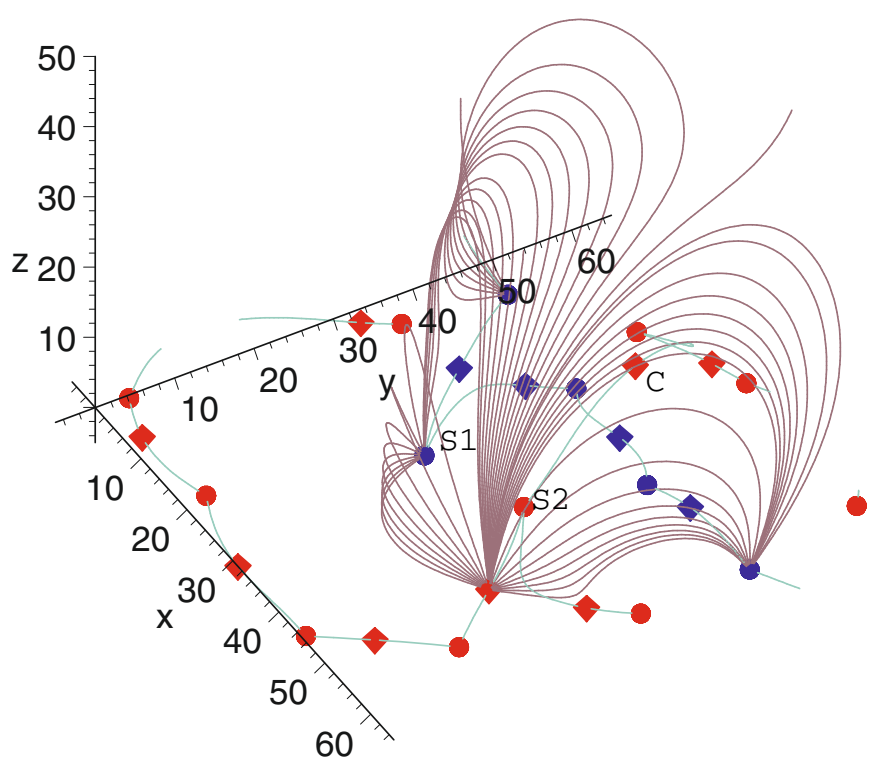

Fig. 9. 3D view of the separatrix surfaces surrounding the rotating positive source, with sources calculated using the 8th-order Fourier-filtering technique.

However, this does not explain why no similar effect was observed in the first simulation. Maybe it existed but was too weak to pick up in the analysis, or maybe it is determined by the detailed geometry of the magnetic field. Another obvious question is what the $3 \mathrm{D}$ distribution of $E_{\|}$looks like; the current work only deals with $E_{\|}$integrated along fieldlines, so it shows which fieldlines are reconnecting, but not at which point along the fieldline the reconnection actually occurs. We hope to do more work towards resolving these issues in the future.

\section{Robustness of topological model}

The details of the magnetic topology that we have been using in this study so far depend on the technique that was chosen to extract the set of point magnetic sources from the magnetogram data. Therefore in this section we consider which features of the topology are robust to changes in the source-calculation technique. To do this, we recalculate the topology under two new sets of assumptions - the smoothed magnetic field and 16th-order Fourier-filtering techniques detailed in Sect. 2.3 - and look for similarities and differences in the results.

\subsection{Smoothed magnetic field sources}

Figure 10 shows the magnetic footprint of the topology based on smoothed magnetic field sources. This technique for selecting sources produces roughly the same number as before; 21 plus the balancing source at infinity, compared with 15 plus one from 8th-order Fourier-filtering. Of these, there are 14 positive sources and 8 negative, including the source at infinity. The distribution of the sources inside the simulation box is broadly similar to that found for 8th-order Fourier-filtered sources. There is a band of positive sources at the top of the box, connected via a line of spines to another concentration of positive sources at the bottom of the box. The negative sources lie more centrally, positioned to both left and right of the previously mentioned line of spines. However, there are a few significant differences. For example, the smoothed magnetic field method places the upper 


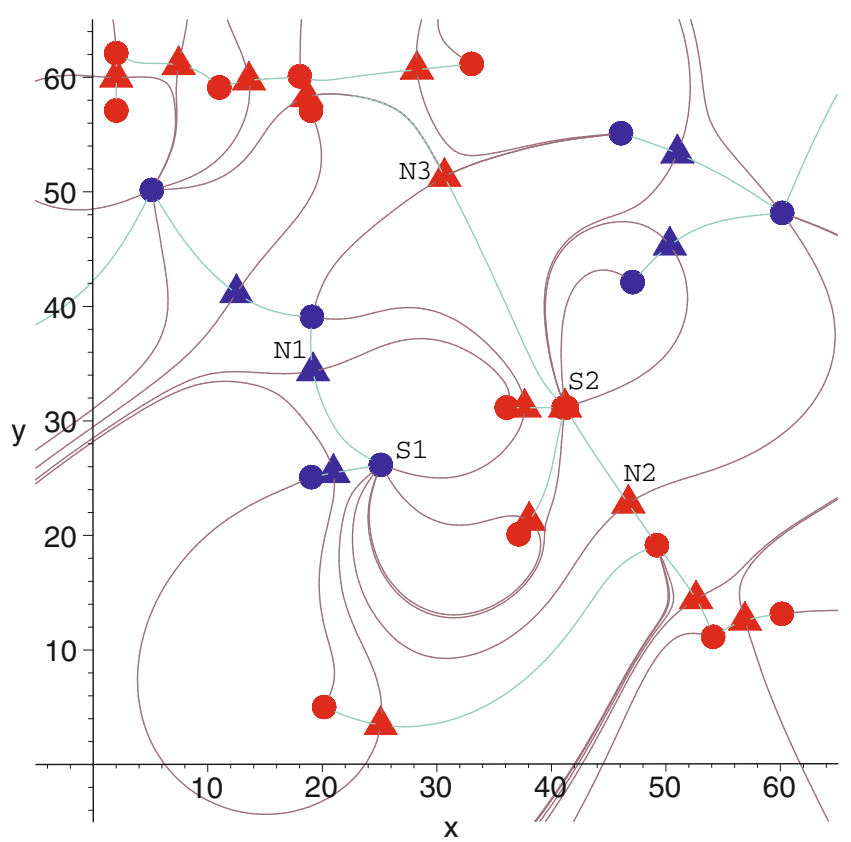

Fig. 10. Photospheric footprint for smoothed magnetic field sources.

positive sources further to the left, but fails to detect the positive sources found on the lower left of the Fourier-filtered footprint.

The new topology contains 13 positive and 7 negative magnetic null points, all of which are prone and photospheric (thus fulfilling the Euler Eqs. (5) and (6)). This contrasts with the previous topology which contains a positive coronal null. It seems that this null still exists in the new topology but has found its way into the photosphere, where it lies at $(31,51)$ and is labelled N3 on the footprint (Fig. 10). It is clear that this is indeed the same null point in both source approximations when you see that its separatrix still forms the same part of the boundary around the main positive source. We will return to this issue later; for now we consider how well this new topology matches the simulation of the negative source rotating.

The separatrix surface of the negative null N1 forms a dome which covers the negative source. It touches the photosphere along its own separatrix traces, one of which leaves the diagram along its lefthand edge. On the other side, the dome continues along the green spine line that runs right through the main positive source and continues diagonally down and right until it encounters the other separatrix trace re-entering the diagram from the lower edge. Figure 11 shows this photospheric boundary of the separatrix dome superimposed on the contour plot of $E_{\|}$integrated along the magnetic fieldlines, to allow easy comparison between the two. 3D views of the dome are given in Fig. 12.

Allowing for the relatively small number of sources in this model and the limitations of using potential fields, there is a good correspondence between the region of high $E_{\|}$integrated along the magnetic fieldlines and the position of parts of the separatrix surface. The separatrix leaves the main positive source at exactly the right angle to coincide with the contours of integrated $E_{\|}$, and turns downwards as they do. Because the smoothed magnetic field approximation fails to pick up some of the positive sources in the lower lefthand corner, there is nothing "pulling" the separatrix towards this corner and so it leaves the diagram at a rather steeper angle than the region of high integrated $E_{\|}$does. Nevertheless, the features of the contour plot are well-explained by current building up along parts of this separatrix dome. Notice

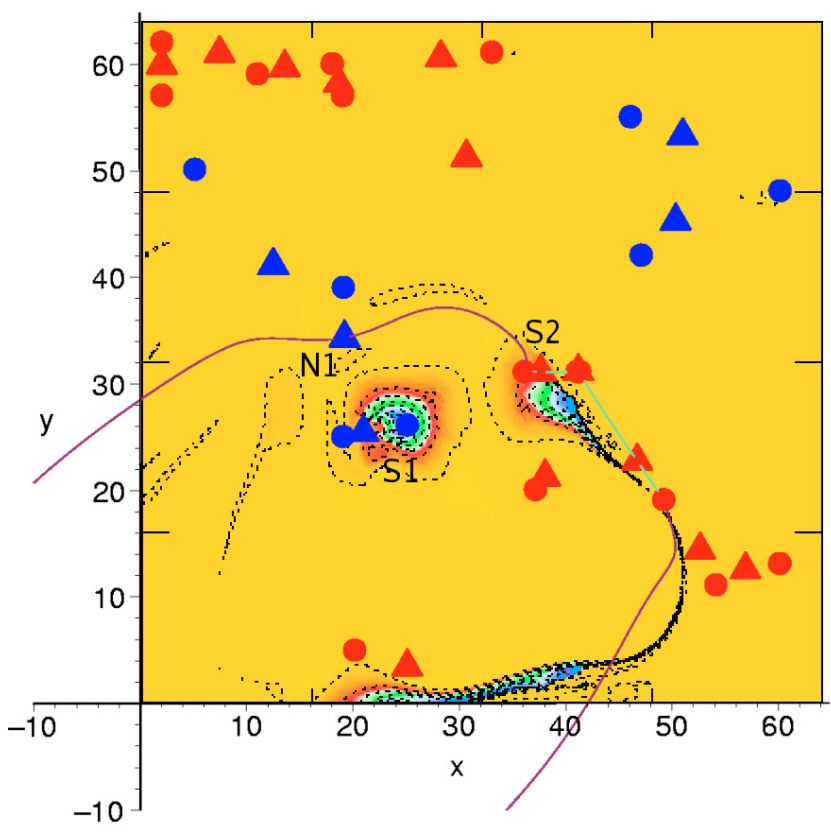

Fig. 11. The photospheric footprint (based on smoothed magnetic field sources) of the separatrix dome that covers the rotating negative source, and how it compares with the contour plot of $E_{\|}$integrated along the magnetic fieldlines.

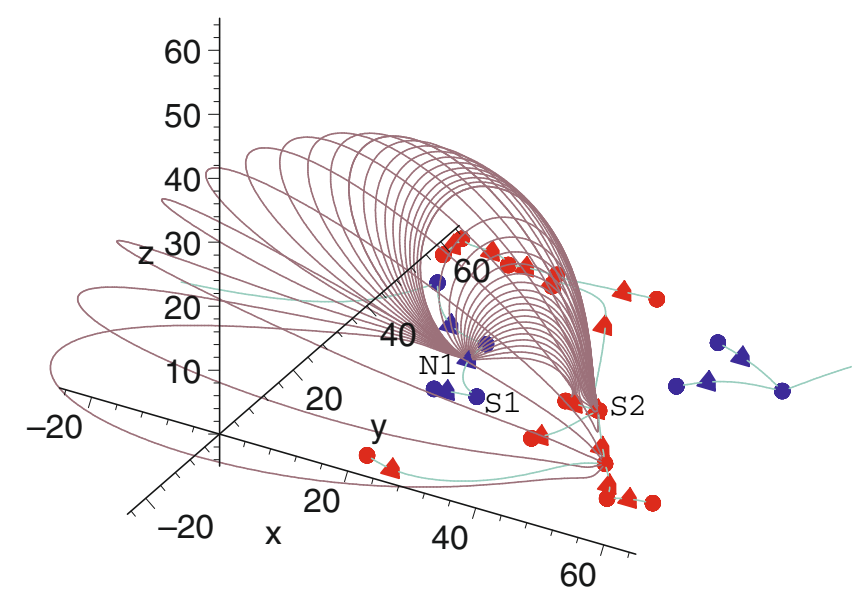

Fig. 12. 3D view of the separatrix dome covering the rotating negative source, with sources calculated using the smoothed magnetic field technique.

also that the negative null producing the dome lies in almost the same position under both source approximations studied so far.

Let's now turn to the equivalent question for the simulation of the main positive source rotating. As in the previous experiment, the positive source is surrounded in the photosphere by the traces of two separatrix surfaces. The null points concerned, $\mathrm{N} 2$ and N3, are both positive, prone, and photospheric. Figure 13 is the usual superposition of these separatrices onto the contour plot of $E_{\|}$integrated along the magnetic fieldlines, for rotation of the positive source.

In the photosphere, each of the two separatrix surfaces in question follows what is in fact the only possible path for it; along its own separatrix traces and then out to each edge of the diagram along the spine lines of the negative nulls. Figure 14 shows $3 \mathrm{D}$ views of these surfaces in the corona. Again, allowing 


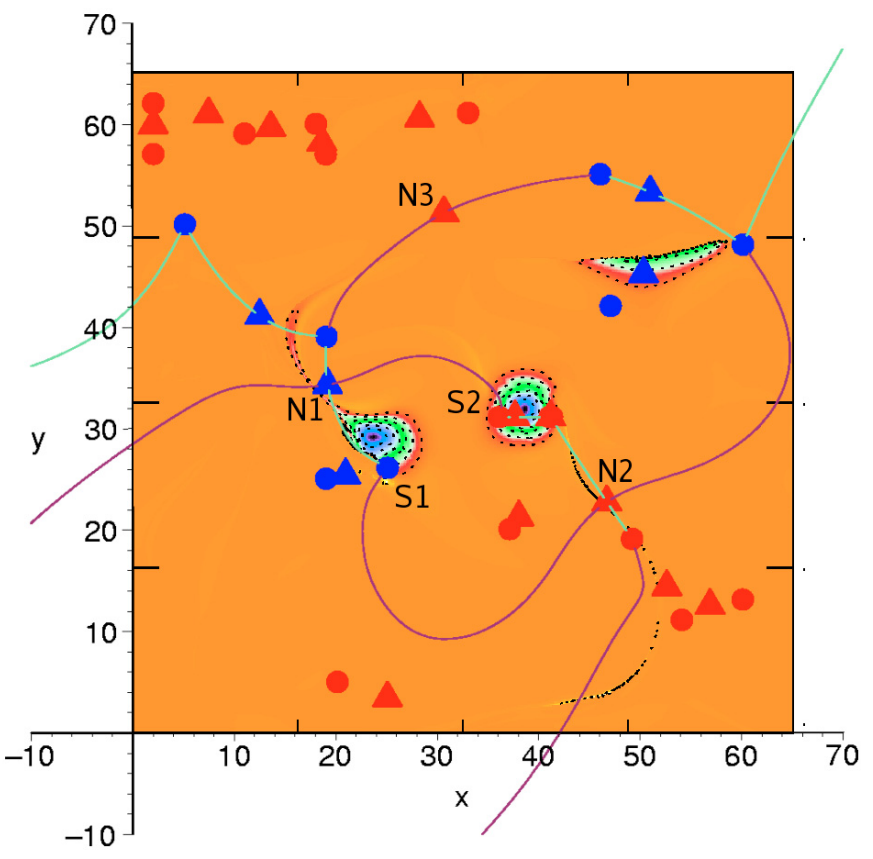

Fig. 13. The photospheric footprint (based on smoothed magnetic field sources) of the separatrix surfaces that surround the rotating positive source, and how it compares with the contour plot of $E_{\|}$integrated along the magnetic fieldlines. Separatrix covering negative source also shown.

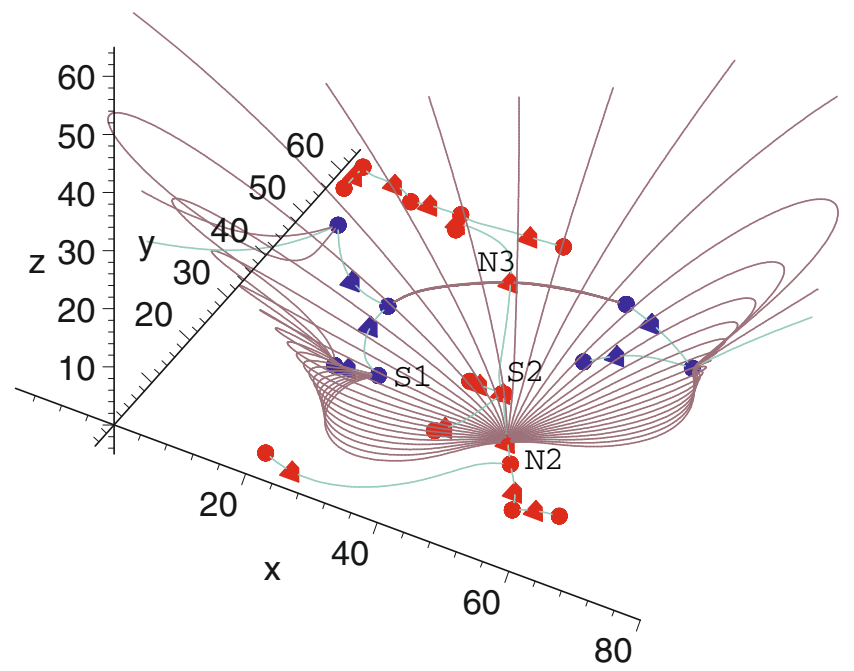

Fig. 14. 3D view of the separatrix surfaces surrounding the rotating positive source, with sources calculated using the smoothed magnetic field technique.

for the small number of sources and the inaccuracies of potential field modelling, the regions of high integrated $E_{\|}$are wellaligned with parts of the separatrix surfaces. In particular, there is an excellent correspondence between parts of the spines leaving the main negative source (which carries the separatrix of the lower positive null) and the region of high integrated $E_{\|}$also connected to the negative source. The region on the top right of the diagram is not so obviously well-correlated with any part of the separatrix surface, but does seem to follow part of the spine of another close-lying negative null. The reason for this may be that the region is close to the edge of the simulation box. The magnetogram shows a large active region directly north of the $\mathrm{BP}$ and about $100^{\prime \prime}$ away from it. This AR is not accounted for

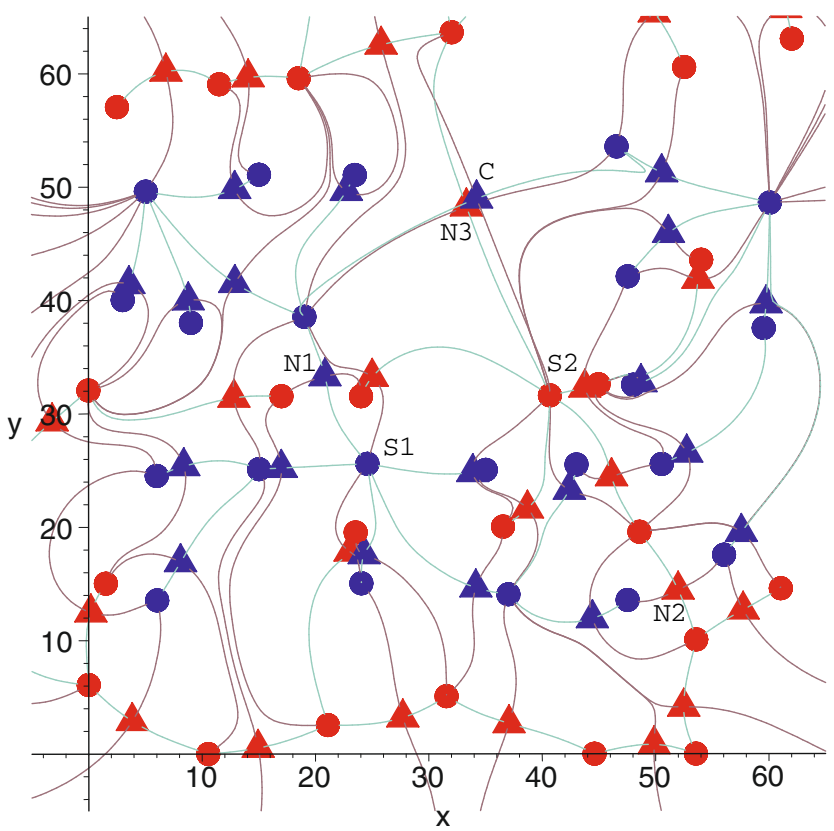

Fig. 15. Photospheric footprint for 16th-order Fourier-filtered sources.

in the model, but its magnetic influence at that edge of the box could force the separatrix of N3 to undergo a bifurcation and lie along the alternative spine path.

\subsection{6th-order Fourier-filtered sources}

The last source approximation that we test here is 16th-order Fourier filtering, as described in Sect. 2.3. 47 sources and 46 null points exist in this topology, which can be seen in Fig. 15. 24 of the sources are positive, and 22 are negative, including the balancing source at infinity. Of the nulls, 24 are positive, and 22 are negative, including one coronal null. These numbers satisfy the Euler Eqs. (5) and (6). With this much larger number of sources than the other two models, this topology looks complicated and crowded at first sight. However, we shall soon find that it contains many familiar features.

The null point N1 whose separatrix covers the rotating negative source can still be picked out. Figure 16 shows the photospheric trace of this separatrix in comparison with the contour plot of $E_{\|}$integrated along the magnetic fieldlines. It follows the line of spines that encloses the main negative source. Clearly, the higher number of sources present in this model leads to a greater accuracy in predicting the locations of high integrated $E_{\|}$ regions found by simulation, as the separatrix sits almost directly over them and in fact the two lines touch and cross several times.

It was not possible to produce $3 \mathrm{D}$ separatrix surface plots for this topology as the null point has such strong eigenvectors in the photospheric plane that coronal fieldlines could not be plotted. However, the structure of the topology requires the separatrix to take the path described above. This is purely an effect of the way in which fieldlines in the separatrix surface are found at present and does not in any way reduce the validity of the findings above. Separatrix surfaces are found by starting fieldlines very close to the null in its fan plane. These fieldlines are usually evenly spaced. However in this case, all the fieldlines show such a strong tendency to close down to the nearest sources that it was impossible to find any that did not, down to the limit of numerical accuracy. This does not mean there is no separatrix 


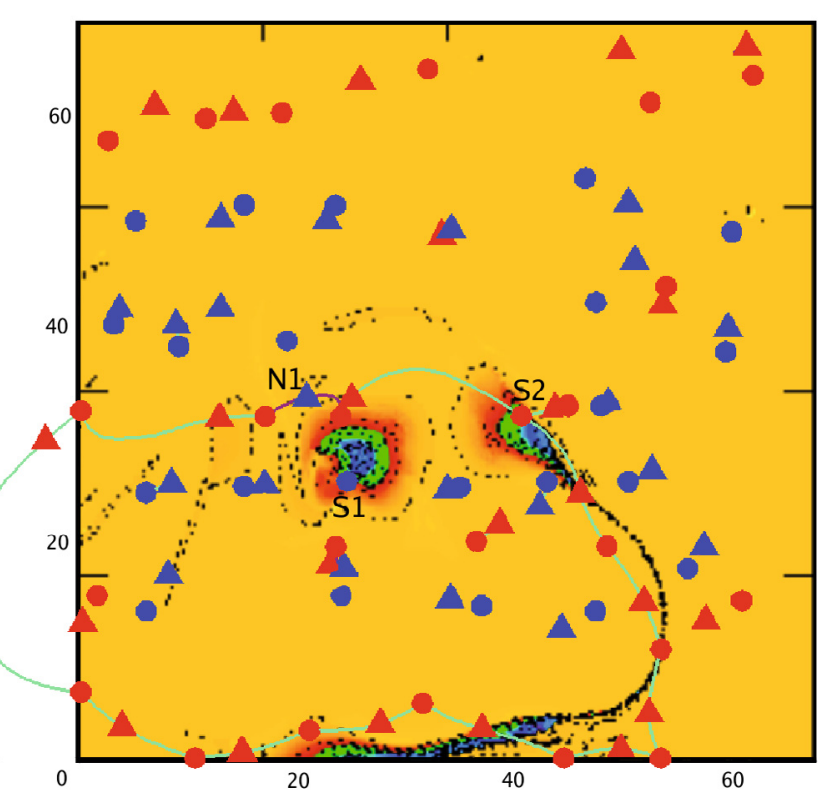

Fig. 16. The photospheric footprint (based on 16th-order Fourierfiltered sources) of the separatrix dome that covers the rotating negative source, and how it compares with the contour plot of $E_{\|}$integrated along the magnetic fieldlines.

though. The separatrix acts as a marker for a change in fieldline connectivity, and this change is still there, just as distinctly as in any other case studied here. Our current methods simply prevent us from detecting any of the fieldlines that mark out this change in this particular case.

For the case of the positive source rotating, as ever, two separatrix surfaces together surround the positive source. These are the separatrices of the positive prone nulls N2 and N3, as shown in Fig. 17, where the positions of the separatrices are compared to the relevant contour plot of integrated $E_{\|}$. In this topology, there is an interesting difference to the other two models. Instead of both separatrices extending out to infinity, each one forms part of a finite dome that actually covers the rotating positive source. The two separatrices meet along the spine line of the negative coronal null point $\mathrm{C}$. So clearly the two positive nulls can be said to be the "same" nulls that were found in the other two models, but the topological shape of their separatrix surfaces (although still fulfilling the same function) has changed.

Comparing the separatrices with the regions of high integrated $E_{\|}$, again the region stretching diagonally up and left from the main negative source is associated with the spine fieldline extending in the same direction that carries the separatrix surface of N3. Although they are not exactly aligned, within the limitations of the model they are undoubtedly related. Concerning the other region, above and to the right of the main positive source, there is the same slight issue as for the smoothed magnetic field model, in that the region seems to follow the wrong spine. Again, we postulate that this may be due to the strong positive active region source which exists in the real solar magnetic configuration but lies outside the simulation box, which could force the separatrix down onto the other spine.

\subsection{Comparison with main nulls in magnetic field from $M H D$ simulation}

The locations of the critical null points associated with the separatrices surrounding the rotating sources are further confirmed

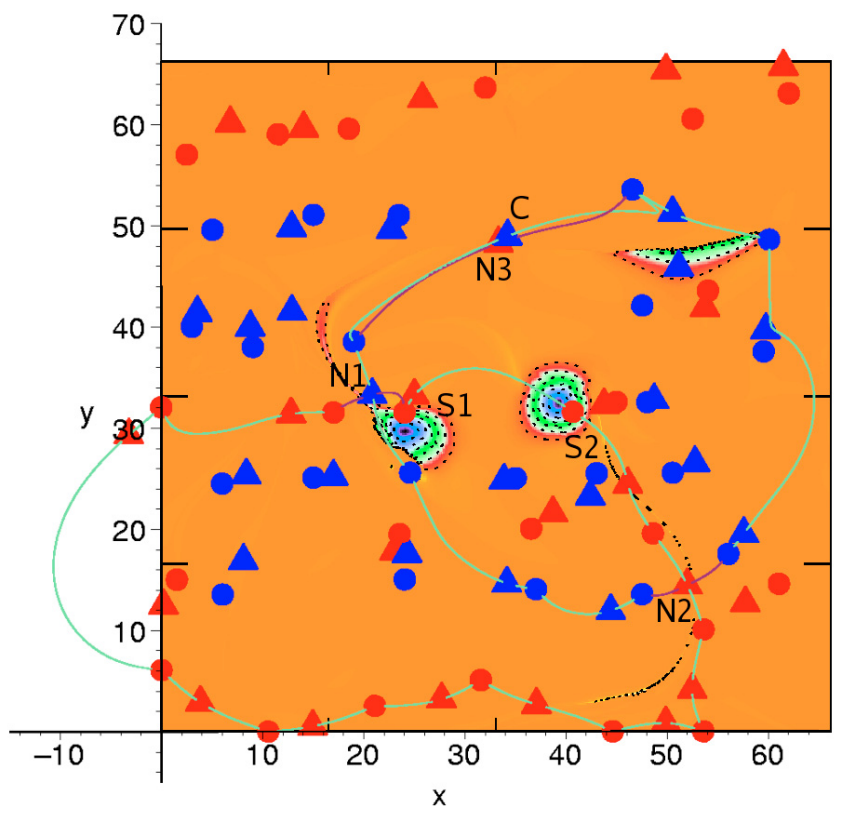

Fig. 17. The photospheric footprint (based on 16th-order Fourierfiltered sources) of the separatrix surfaces that together cover the rotating positive source, and how it compares with the contour plot of $E_{\|}$ integrated along the magnetic fieldlines. Separatrix covering negative source also shown.

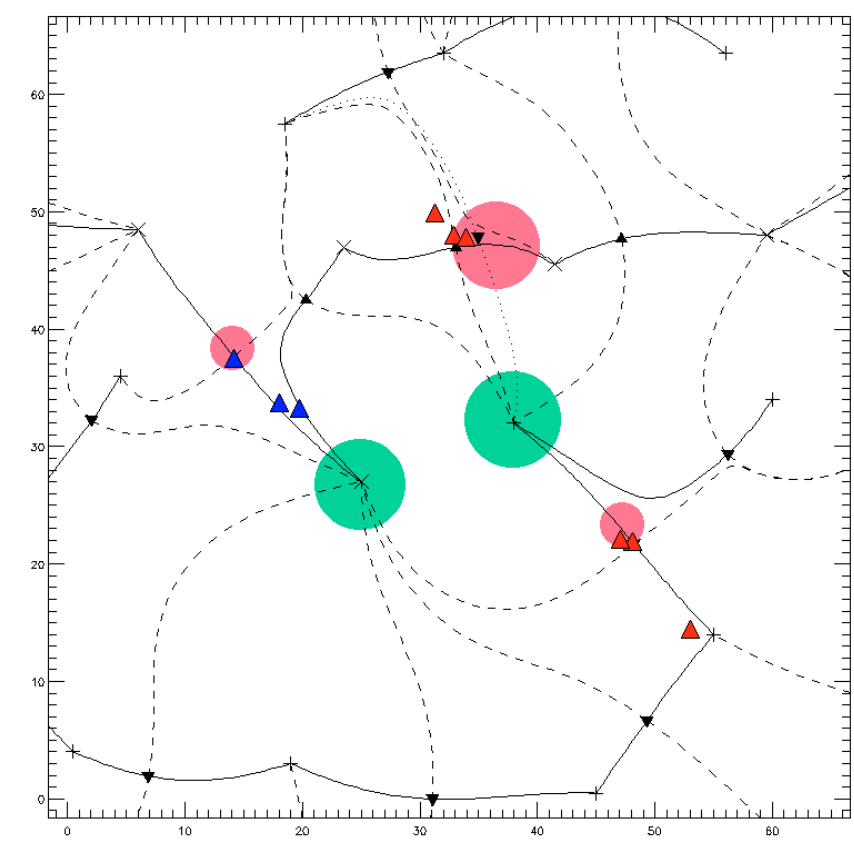

Fig. 18. Positions of main nulls in the magnetic field from the MHD simulation. The areas containing the main sources are large green circles and the areas containing the main nulls are large red circles. We have superimposed red and blue triangles showing the locations of the critical nulls from the three topological reconstructions of the region.

by another piece of analysis of the bright point region. Figure 18 shows the approximate positions of the three main nulls found in the magnetic field produced by the MHD simulation discussed in Sect. 2.1, by searching for regions of minimum field where $|\boldsymbol{B}|$ is close to zero. This technique uses the whole $3 \mathrm{D}$ field to work out where null points are likely to be found, although it does not give their exact positions. We have superimposed on the plot 


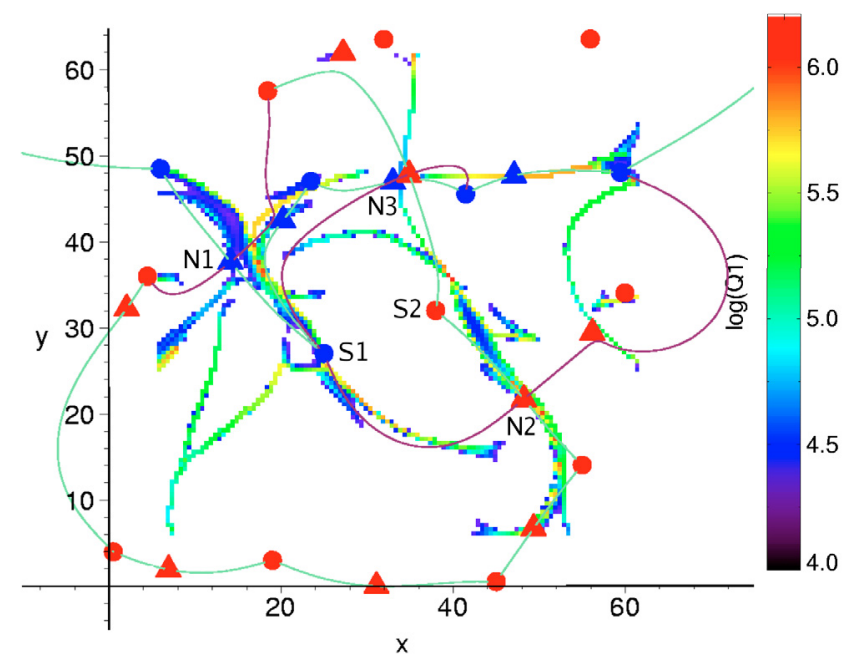

Fig. 19. QSLs in the magnetic field from the MHD simulation. Blue corresponds to regions of high squashing factor. We have superimposed the separatrices from the 8th-order Fourier-filtered topological model of the bright point region.

the positions of the three magnetic nulls $(\mathrm{N} 1, \mathrm{~N} 2$, and N3/C) whose separatrices cover the rotating sources, from all three of our topological reconstructions of the bright point region.

It can be seen from Fig. 18 that five of the nine nulls from the topological reconstructions overlap with the regions of low $|\boldsymbol{B}|$. The remaining four do not show quite such a good correspondence but there is a definite trend towards their being located near the null point regions. This provides a useful confirmation that the nulls selected as being topologically important in the analysis performed in previous sections are likely to be the main null points of the region, and that their locations can be identified by an alternative technique.

\subsection{Comparison with calculated locations of QSLS from MHD simulation}

The positions of the separatrices are also important as it is at certain locations along these surfaces that strong currents are generated so that as a result of plasma instabilities that cause anomalous resistivity, reconnection can take place (Büchner 2006b). All the separatrices in the topological reconstructions are calculated for the case of a potential field, so it is useful to know how the positions of these separatrices correspond to the positions of the quasi-separatrix layers in the magnetic field from the MHD simulation. The QSLs were calculated numerically and are shown in Fig. 19. Also in this figure we have superimposed the photospheric traces of the separatrices from the 8th-order Fourier-filtered topological model of Sect. 3.

There is a reasonably good correspondence between the two. The footprints of the domes surrounding both rotating sources can be clearly made out in the QSL plot. Particularly close correlations occur along the line of spines of the negative nulls, that bounds the separatrix of the coronal null, and also along the lower righthand edge of the main negative source's separatrix dome. Similar comparisons for the smoothed magnetic field and 16th-order Fourier-filtered cases can be made as well, and also show a convincing correlation to the locations of the QSLs. Of course, there are certain regions where the correspondence is not so good, but these can be explained as before by edge effects or simply by not enough sources having been included in the topological model to produce sufficient accuracy.

A quick comparison by eye shows that there is, as expected, almost perfect alignment of the QSLs with the locations of high $E_{\|}$integrated along the magnetic field, from Figs. 4 and 7. The high level of agreement between the locations of potential separatrices and MHD QSLs is good evidence that potential field modelling is able, even in the presence of currents in the MHD simulations, to make accurate predictions about the locations of reconnection events, up to a certain accuracy. We have shown that MCT modelling can predict which separatrix surface will host the reconnection, and can even predict the shape of that separatrix with reasonable success. However, the obvious question remaining is, why does only part of the separatrix surface correspond to the location of the strong integrated parallel electric field and the QSL? Clearly there is still another factor at play here which we cannot account for at present, although we have tentatively suggested in Sect. 4.1 that the QSLs are primarily located close to the strong photospheric magnetic source regions, and become spread further along the separatrix surface boundary due to rotation of the sources.

\section{Comparison with TRACE observations}

The TRACE satellite observed the bright point region during its $\pi$-phase, just after the rotation phase. Figure 20 shows an observation in the 171 A passband from 14:02:30 UT. Most of the emission is concentrated near the two main magnetic polarities of the bright point, as well as at the strong magnetic sources at the top left and bottom centre of the observed region.

The contours of the integrated parallel electric field (from the numerical simulations described earlier) have been overlaid onto the TRACE observations. Visually, there seems to be a good correspondence between the areas of strong emission in the TRACE images and the areas where fieldlines with high values of integrated parallel electric field are rooted. This lends weight to the theory that magnetic reconnection is powering the emission from the bright point.

However, the regions of high $E_{\|}$integrated along the magnetic field and the regions of strong $171 \AA$ emission observed by TRACE are not entirely cospatial. There are a few discrepancies, such as the integrated electric currents at the lefthand side of the BP, the bright region on the left that extends further north than the strong integrated electric currents, and the general shape of the observed BP. The shape does seem to be matched more closely by some portions of the calculated QSLs than by the high integrated $E_{\|}$regions, although the QSLs are not thick enough to cover the whole observed brightenings. We believe that these deviations may be caused by other photospheric motions not included in our model (which only looked at the rotation of one BP magnetic source region) or by the influence of the strong magnetic field from the nearby AR (see Sect. 5.1).

\section{Discussion}

In this work we topologically model the solar atmospheric magnetic field in a region where an EUV bright point was observed in the corona, and compare the results with 3D numerical MHD simulations of rotating the two main photospheric magnetic sources that underlie the bright point. We find that both of the sources are associated with separatrix surfaces that completely surround them in the photosphere. Rotating a source causes large 

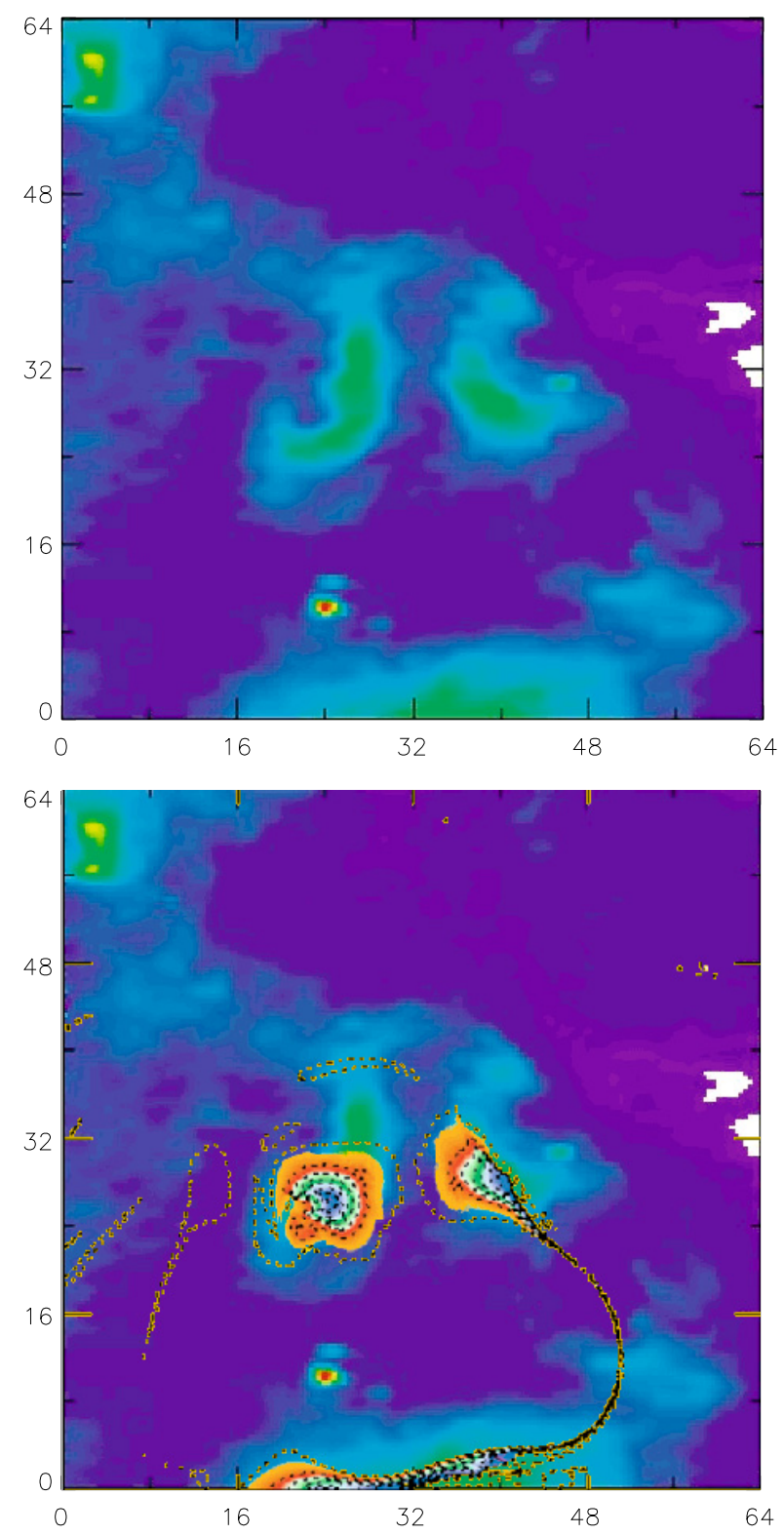

Fig. 20. Above: TRACE $171 \AA$ observation of the bright point at 14:02:30 UT, after the rotation phase. Brighter regions are shown in green, dimmer in blue, with the same field of view as the earlier figures. This TRACE passband mainly shows up brightenings in the transition region, not coronal loops. Below: the same TRACE observation, with overlaid contours in orange, red, green, and blue showing the values of the parallel electric field integrated along the magnetic fieldlines, from the numerical simulation.

concentrations of parallel currents to build up and, after they exceed a plasma-physical threshold, electric fields can build up in the region that are well-correlated with the positions of the separatrix surfaces.

This means that the shear caused by the rotation of a source in turn causes current buildup, that can be dissipated causing reconnection and plasma heating on the separatrix surfaces associated with that source. Hence, potential magnetic field topological models can provide reasonably accurate predictions of locations where enhanced currents and reconnection might be expected to occur.

We use three different approximations to derive the starting set of point magnetic sources from the continuous magnetogram data: 8th- and 16th-order Fourier filtering, and smoothing the magnetic field. Each approximation leads to a different set of sources, so the aim was to find out how robust the large-scale topology is to these changes. In fact, we find that some elements of the topology are remarkably robust. The null points studied here, whose separatrices are large compared to the average distance between null points, can easily be identified in all three cases. Of course, as the number of sources in the model increases, its accuracy in predicting reconnection sites also increases, except for sites close to the edge of the simulation box which can be affected by sources from outside the box not included in the model.

Despite the widely-held belief that coronal nulls are more robust than their photospheric counterparts, we do not find this to be true for the bright point topology studied here. One case includes a positive coronal null which is photospheric in the other two cases, one case has no coronal nulls, and the last has a negative coronal null with no obvious counterpart in the first two cases. However, we do find that the topology preserves its essential large-scale character, independently of the precise method used to select the source set. This is a welcome validation of the magnetic topology technique, as it is specifically intended to detect and model the large-scale pattern of magnetic connections between source regions. It is not related to the complexity of the field studied here; indeed, it might be expected that the choice of sources would have more impact in a region such as this without an overwhelmingly-strong dominant source pair.

The large-scale topology is independent of the method of choosing source regions (within reason!) because it reflects the real topological properties of the real magnetic field. This is also why the MCT model is in close agreement with the QSL model on the location of the (quasi-)separatrices. However, this should not be taken to imply that the choice of magnetic source regions has no effect at all. The small-scale topology shows fluctations depending on the choice of sources. These fluctuations are artefacts of the model. The results of an MCT reconstruction are only reliable on a large scale compared to the source separation, which is fortunately exactly the scale that interests us in this work.

Comparison of the locations of the nulls and separatrices in a potential field with the nulls and QSLs from the MHD simulations shows that in general, and despite its exclusion of currents, the magnetic topology derived from the extrapolated potential field can be used to determine the locations of the separatrix surfaces along part of which the current concentrations linked to reconnection and BP heating will build up. Further work is required to precisely explain why only certain portions of the separatrix surface coincide with regions of high integrated parallel electric field, as the MCT model cannot predict or explain this. A comparison with the observational data from TRACE showed a strong correlation between the locations of brightenings and the locations where fieldlines with high values of integrated parallel electric field are rooted in the photosphere.

For future work, it would be very interesting to test these results on a wider range of observed coronal bright points, to find out whether the results are general or specific to the case studied here.

Acknowledgements. J. Büchner, R. Maclean and E. Priest gratefully acknowledge the opportunities for interaction provided by the Isaac Newton Institute MRT Workshop in Cambridge, August 2004 and the Workshop on Magnetic Reconnection in Florence, September 2006 as well as of the DAAD-ARC project ARC D/05/26090. R. Maclean is grateful for financial support from the UK Science and Technology Facilities Council. We also thank Gunnar Hornig for helpful discussion and suggestions, and the European Commission for financial 
support through the SOLAIRE Network (MTRN-CT-2006-035484). J. Büchner would also like to acknowledge the stimulating discussions in the ISSI team lead by G. Poletto on Role of current sheets in solar eruptive events supported by the International Space Science Institute (ISSI) in Bern.

\section{References}

Brown, D. S., \& Priest, E. R. 1999, Sol. Phys., 190, 25

Brown, D. S., \& Priest, E. R. 2000, Sol. Phys., 194, 197

Brown, D. S., Parnell, C. E., Deluca, E. E., Golub, L., \& McMullen, R. A. 2001 Sol. Phys., 201, 305

Büchner, J. 2006a, Space Sci. Rev., 122, 149

Büchner, J. 2006b, Space Sci. Rev., 124, 345

Büchner, J. 2007, in New Solar Physics with Solar-B Mission, ed. K. Shibata, S. Nagata, \& T. Sakurai, ASP Conf. Ser., 369, 407

Büchner, J., \& Elkina, N. 2006, Phys. Plasmas, 13

Büchner, J., \& Elkina, N. V. 2005, Space Sci. Rev., 121, 237

Büchner, J., \& Daughton, W. 2007, in Reconnection of Magnetic Fields: Magnetohydrodynamics, Collisionless Theory and Observations, ed. J. Birn, \& E. Priest (Cambridge, UK: Cambridge University Press), 144

Büchner, J., Nikutowski, B., \& Otto, A. 2004, in SOHO 15 Coronal Heating, ed. R. W. Walsh, J. Ireland, D. Danesy, \& B. Fleck, ESA SP-575, 35
Büchner, J., Nikutowski, B., \& Otto, A. 2005, in Astrophysical Particle Acceleration in Geospace and Beyond, ed. D. Galagher, \& J. Horwitz (Washington DC.: AGU monograph series), 272

Démoulin, P., Priest, E. R., \& Lonie, D. P. 1996, J. Geophys. Res., 101, 7631

Golub, L., Krieger, A. S., Harvey, J. W., \& Vaiana, G. S. 1977, Sol. Phys., 53, 111

Longcope, D. W. 1996, Sol. Phys., 169, 91

Otto, A., Büchner, J., \& Nikutowski, B. 2007, A\&A, 468, 313

Parnell, C. E., Smith, J. M., Neukirch, T., \& Priest, E. R. 1996, Phys. Plasmas, 3, 759

Priest, E. R., \& Démoulin, P. 1995, J. Geophys. Res., 100, 23443

Priest, E. R., \& Titov, V. S. 1996, Phil. Trans. Roy. Soc. A, 354, 2951

Priest, E. R., Parnell, C. E., \& Martin, S. F. 1994, ApJ, 427, 459

Priest, E. R., Longcope, D. W., \& Heyvaerts, J. 2005, ApJ, 624, 1057

Shay, M. A., Drake, J. F., Denton, R. E., \& Biskamp, D. 1998, J. Geophys. Res., 103,9165

Titov, V. 1999, in Magnetic Fields and Solar Processes, ed. A. Wilson et al., ESA SP-448, 715

Titov, V. S., Hornig, G., \& Démoulin, P. 2002, J. Geophys. Res. (Space Physics), 107,3

Vaiana, G. S., Krieger, A. S., Speybroeck, L. P., \& Zehnpfennig, T. 1970, Bull. Am. Phys. Soc., 15, 611

Vernazza, J. E., Avrett, E. H., \& Loeser, R. 1981, ApJS, 45, 635

Yokoyama, T., \& Shibata, K. 1996, Pub. Astron. Soc. Japan, 48, 353 